\title{
Management of Patients with Relapsed and/or Refractory Multiple Myeloma Treated with Novel Combination Therapies in Routine Clinical Practice in Germany
}

\author{
H. Tilman Steinmetz (D) - Moushmi Singh · Joseph Milce • \\ Mohamad Haidar · Achim Rieth · Andrea Lebioda · Jörn Kohnke
}

Received: October 30, 2021 / Accepted: December 15, 2021 / Published online: January 16, 2022

(C) The Author(s) 2022

\begin{abstract}
Introduction: Multiple myeloma remains an incurable plasma cell malignancy which, despite improvements in overall survival over the last decade, is characterized by recurrent relapse and is associated with a poor prognosis. This study investigates the use of novel agents in current real-world clinical practice in the management of relapsed and/or refractory multiple myeloma (RRMM) in Germany over different lines of therapy.

Methods: A retrospective chart review was conducted for patients with RRMM treated at multiple centers across Germany between May 2017 and June 2018. Variables included patient
\end{abstract}

Supplementary Information The online version contains supplementary material available at https:// doi.org/10.1007/s12325-021-02022-z.

H. T. Steinmetz $(\bowtie)$

Center for Hematology and Oncology, Oncology Cologne, Sachsenring 69, 50677 Cologne, Germany

e-mail: steinmetz@oncokoeln.de

M. Singh

Amgen Ltd, Uxbridge, UK

J. Milce $\cdot$ M. Haidar

Kantar Health, Paris, France

A. Rieth · A. Lebioda

Amgen GmbH, Munich, Germany

J. Kohnke

Onkologie Zentrum Sud, Remscheid, Germany demographics and clinical characteristics, current and prior treatment regimens, treatment response, cytogenetic abnormalities, testing methodology, and resource utilization.

Results: Data were analyzed from 484 patients from 47 centers across Germany (60\% male; average age over 70 years; majority at International Staging System stage 2 or 3). Bone pain and anemia were the most common symptoms at diagnosis, with $63 \%$ of patients receiving osteoprotective drugs. Approximately one-third (32\%) of patients had received autologous stem cell transplantation and approximately 70\% underwent cytogenetic testing. After failure to respond to first-line treatment, most patients received regimens containing second-generation proteasome inhibitors and monoclonal antibodies, with overall response rates greater than $90 \%$ in second line $(95 \%$ and $90 \%$ for daratumumab-based and carfilzomib-based therapies, respectively). The incidence of unplanned hospitalization ranged from $11 \%$ to $16 \%$ across all treatment lines, with longer hospital stays required for treatment administration than for treatment-related toxicity.

Conclusion: Although treatment patterns for RRMM in Germany differ by line of therapy and are adapted as disease progresses, patients mostly receive combination regimens with carfilzomib or daratumumab in second and third lines, with high overall response rates achieved in all lines. 
Keywords: Carfilzomib;

Daratumumab; Germany; Monoclonal antibody; Proteasome inhibitor; Real-world; Recurrent/refractory multiple myeloma; Treatment patterns

\section{Key Summary Points}

Why carry out this study?

Multiple myeloma is characterized by recurrent relapse and is associated with a poor prognosis, despite improvements in overall survival over the last decade.

This multicenter chart review examines the use of novel agents in the real-world management of relapsed and/or refractory multiple myeloma (RRMM) across different lines of therapy in Germany.

Variables included patient demographics and clinical characteristics, current and prior treatment regimens, treatment response, cytogenetic abnormalities, testing methodology, and resource utilization.

\section{What was learned from this study?}

Although treatment patterns differ by line of therapy and are adapted as disease progresses, patients mostly receive combination regimens with carfilzomib or daratumumab during second- and third-line treatment, with high overall response rates achieved in all lines.

Despite the large proportion of high-risk patients, the percentage of patients in remission was slightly higher (75-78\%) than that reported in other analyses (66-73\%).

\section{INTRODUCTION}

Multiple myeloma is a plasma cell malignancy that accounts for approximately $1 \%$ of all neoplasms and $10 \%$ of all hematologic malignancies [1]. In Europe, age-standardized incidence rates per 100,000 people were estimated to be
4.7 for men and 3.1 for women in 2012 [2], while in Germany, the age-standardized incidence rates have been estimated to be 6.1 for men and 3.8 for women [3]. Incidence rates increase steadily with age, with a peak agespecific incidence rate of 49 per 100,000 in people over 80 years of age [4].

Multiple myeloma remains an incurable malignancy which, despite progressive improvements in overall survival over the last decade, is characterized by recurrent relapse with progressively shorter duration of response and treatment-free intervals with each subsequent treatment [5]. The severity of multiple myeloma can be measured using routinely obtained laboratory tests and assessed using the International Staging System (ISS) [6]. The ISS is a powerful and reproducible three-stage classification that relies on the combination of serum levels of $\beta_{2}$-microglobulin, representing tumor mass and renal function, and albumin, reflecting inflammatory cytokines, with ISS stage III associated with the poorest outcome [7].

In addition, another key variable that affects treatment strategy is risk stratification of patients into standard and high-risk disease. High-risk multiple myeloma is defined by the presence of $\mathrm{t}(4 ; 14), \mathrm{t}(14 ; 16), \mathrm{t}(14 ; 20)$, gain $1 \mathrm{q}$, del(17p), or p53 mutations, which confer poor prognosis and may impact the choice of therapy [8].

Decisions concerning whether or not autologous stem cell transplantation (ASCT) can be considered as part of the treatment strategy depend on a number of factors, including age, comorbidities, cognitive and physical functioning, and the biology of multiple myeloma. For those patients in good health and eligible for ASCT, standard treatment consists of four to six courses of induction with three drug regimens, followed by high-dose melphalan therapy with ASCT [9]. Following ASCT, maintenance therapy with immunomodulatory drugs (IMiDs) such as lenalidomide, or in very advanced stage disease, pomalidomide, is recommended [9]. Owing to the increasing number of new drugs/combinations, several options are available for first-line elderly, frail, or transplant-ineligible patients [9], as well as for patients receiving second-line therapy [10]. 
Thus, decisions concerning the optimal secondor later-line therapies depend on the efficacy, duration of response, and side effects of firstline therapy, as well as the patient characteristics.

Over the past 15 years, several new drugs have been introduced for the treatment of multiple myeloma. These include proteasome inhibitors (carfilzomib, ixazomib, and bortezomib) and monoclonal antibodies (daratumumab, elotuzumab, and isatuximab), which are approved for use either as monotherapy and/or in combination [11], as well as pomalidomide and panobinostat, both of which are used in combination with bortezomib and/or dexamethasone. These are also the most commonly used regimens in relapsed and/or refractory multiple myeloma (RRMM) $[12,13]$, with many patients receiving five or more lines of therapy in a sequential manner over several years.

However, despite the multiple treatments which are available that can now prolong remission, the prognosis of multiple myeloma is poor, with an approximate 56\% 5-year survival rate [14], although survival can be as long as 20 years in some patients. Therefore, treating multiple myeloma is a challenge and finding the appropriate strategy to prolong survival is key. Furthermore, effective treatments in multiple myeloma are needed to delay disease progression, achieve prolonged remission, and thereby preserve quality of life. This was recently shown in a real-world study of patients with multiple myeloma in Germany, which demonstrated that their quality of life was negatively impacted and that quality of life also decreased with increasing lines of therapy [15]. The aims of the current study were to describe the real-world treatment patterns of patients with RRMM in clinical practice in Germany, as well as to gain an understanding of the patient characteristics and other factors that determine treatment choice.

\section{METHODS}

\section{Study Design}

This was a national, multicenter, retrospective medical chart review of patients with RRMM treated at participating centers in Germany between October 2019 and February 2020. This study focused on the use of novel therapies and was conducted in hospitals and office-based centers. Participating hematologists or oncologists must have had at least 3 years of clinical experience, must have been personally responsible for managing and treating symptomatic patients with multiple myeloma, and must have been able to provide at least six medical records of patients across second and third lines of treatment, and at least one patient on fourthline treatment, all of whom had received at least one dose of the pre-specified regimens during the eligibility period. The selection criteria included patients with multiple myeloma who were aged 18 years or over, who had at least one relapse from prior therapy, and met one of the following treatment criteria:

- Patients receiving second- or third-line multiple myeloma treatment who had at least one dose of KRd (carfilzomib-lenalidomide-dexamethasone), Kd (carfilzomib-dexamethasone), DRd (daratumumablenalidomide-dexamethasone), DVd (daratumumab-bortezomib-dexamethasone), or lenalidomide as part of a combination therapy (other than the aforementioned lenalidomide-containing regimens), or bortezomib as part of a combination therapy (other than the aforementioned bortezomibcontaining regimens).

- Patients receiving fourth- or later-line multiple myeloma treatment who had at least one dose of $\mathrm{KRd}, \mathrm{Kd}$, daratumumab monotherapy, or pomalidomide-based combinations during the study eligibility period, irrespective of the treatment starting date. 


\section{Data Collection}

In order to include a minimum of 350 patients across Germany, the study planned to involve approximately 50 physicians/centers, each of whom was able to abstract data for up to seven consecutive patients. It was planned that each physician collects, on average, approximately $80 \%$ second- and third-line data and 20\% fourth- or later-line data. The sample size was not powered for treatment comparisons and this study aimed to provide a purely descriptive review of the clinical practice and outcomes of patients, based on the inclusion criteria and experience from previous studies in multiple myeloma in Germany $[12,16]$.

Physicians documented patients who received either second- or third-line multiple myeloma treatment between October 1, 2017 and June 30, 2018, and patients who received fourth- or later-line treatment between May 1, 2017 and June 30, 2018. The medical records were extracted between October 9, 2019 and February 22, 2020. Outcome variables included patient demographics and clinical characteristics at diagnosis and on enrollment into the study, as well as details of treatment regimen at current line and previous lines of therapy, treatment response, and exploratory minimal residual disease (MRD) testing methodology and prevalence. In addition, details of resource utilization were documented, including hospitalizations (type, number, and length of stay), number of outpatient visits, related medications (e.g., growth factors or transfusions), scans, or other procedures.

\section{Data Analysis}

This was a retrospective descriptive study, no formal hypothesis was tested and, therefore, no formal inferential statistical tests were performed. However, exploratory significance testing was performed using the chi-square test and the resultant $p$ values were included for completeness. Data were stratified into carfilzomibbased regimens (including $\mathrm{Kd}$ and KRd), daratumumab-based regimens (including DRd and DVd), and lenalidomide- and bortezomib-based combinations, and by treatment line. Treatment response was assessed by the treating physicians, based on the International Myeloma Working Group (IMWG) uniform response criteria for multiple myeloma $[17,18]$. Healthcare resource utilization was captured in the form of planned and unplanned medical resource use (i.e., hospitalizations). Categorical/ ordinal variables were described by counts and proportions of respondents, and continuous numerical variables were described by their means and standard deviations, or medians (95\% confidence interval or first and third quartile). Patients with missing data or with no resource use reported, i.e., zero hospitalization, were excluded from mean/median calculations.

\section{Compliance with Ethics Guidelines}

This study was conducted retrospectively from data obtained for clinical purposes and complied with all relevant market research guidelines and legal obligations [19]. No identifiable protected health information was extracted during the study, and we consulted with the institutional review board (IRB) of Nordrhein (Düsseldorf) who determined that our study did not need ethical approval owing to the anonymization of the data collected. An official waiver of ethical approval was granted from the IRB of Nordrhein (Düsseldorf) on July 1, 2019.

\section{RESULTS}

A total of 515 physicians were contacted, of whom 47 hematologists/oncologists from 47 centers across various regions of Germany (West 23; South 17; East 6; North 1) agreed to participate. Data were abstracted from medical records of 484 patients, including 214 (44\%) on second-line therapy, 160 (33\%) on third line, and $110(23 \%)$ in the fourth- or later-line setting. The median overall times from first- to second- and first- to third-line therapy were 12.0 (interquartile range [IQR] 3.0; 26.0) months and 29.0 (IQR 20.0; 40.0) months, respectively. The median (IQR) times between first and second, second and third, and third and fourth lines of treatment were 12.0 (3.0; 


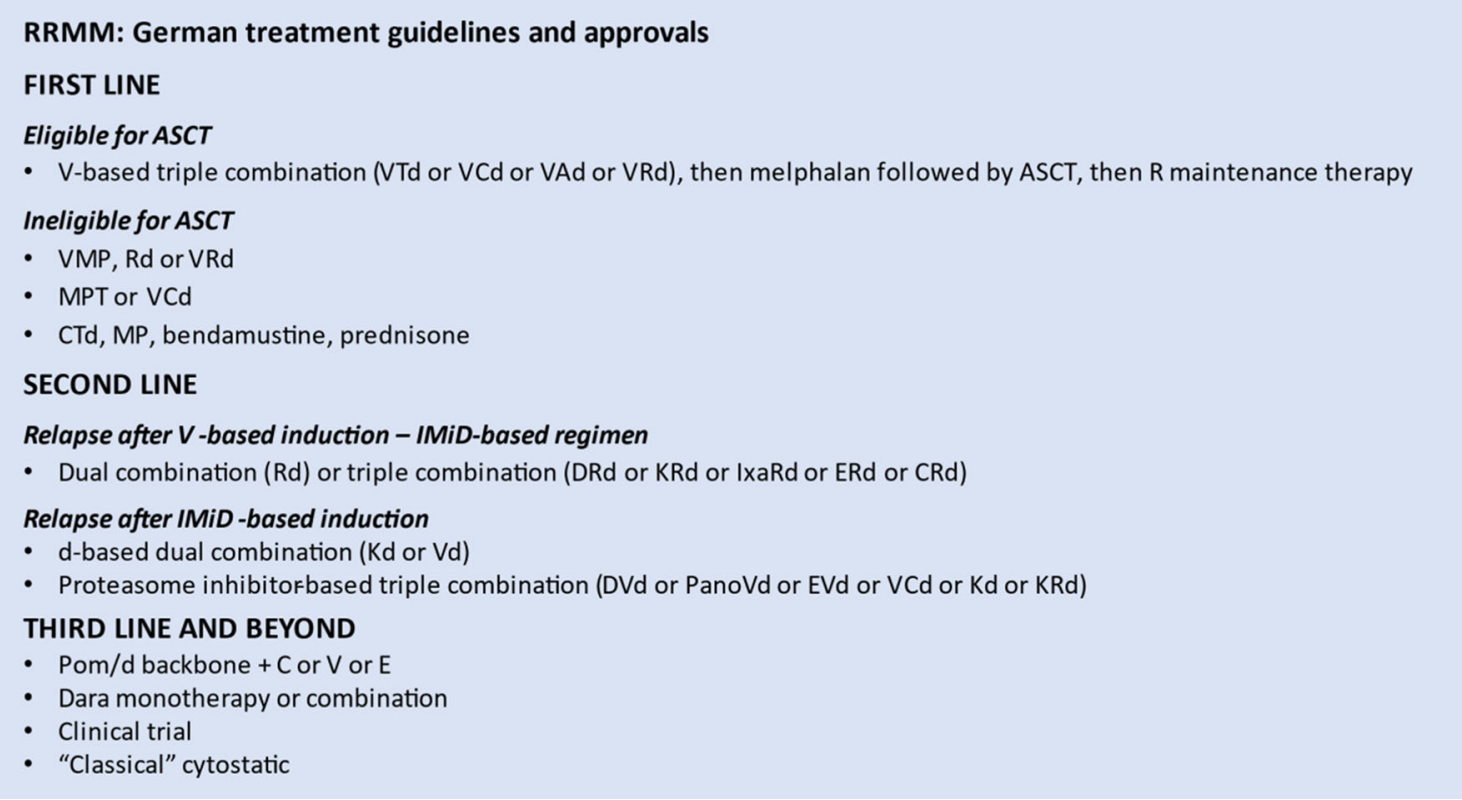

Sources: https://www.onkopedia.com and ESMO Clinical Practice Guidelines [9]. ASCT autologous stem cell transplant, $C$ cyclophosphamide, CRd cyclophosphamide-lenalidomide-dexamethasone, CTd cyclophosphamide-thalidomide-dexamethasone, $d$ dexamethasone, $D R d$ daratumumab-lenalidomide-dexamethasone, $D V d$ daratumumab-bortezomib-dexamethasone, $E$ elotuzumab, $E R d$ elotuzumab-lenalidomide-dexamethasone, $E V d$ elotuzumab-bortezomib-dexamethasone, IMiD immunomodulatory drug, IxaRd ixazomib-lenalidomide-dexamethasone, $K d$ carfilzomib-dexamethasone, KRd carfilzomib-lenalidomide-dexamethasone, $M M$ multiple myeloma, $M P$ melphalan-prednisone, $M P T$ melphalan-prednisone-thalidomide, PanoVd panobinostat-bortezomib-dexamethasone, Pom pomalidomide, $R$ lenalidomide, $R d$ lenalidomide-dexamethasone, $V$ bortezomib, $V A d$ bortezomib-doxorubicin-dexamethasone, $V C d$ bortezomib-cyclophosphamide-dexamethasone, $V d$ bortezomib-dexamethasone, $V M P$ bortezomib-melphalan-prednisone, VRd bortezomib-lenalidomide-dexamethasone, $V T d$ bortezomib-thalidomide-dexamethasone

26.0), $1.0(0.0 ; 2.0)$, and $8.0(5: 0 ; 12.0)$ months (Fig. 1). The number of patients receiving carfilzomib, daratumumab, and other combinations at each treatment line is shown in Table 1. The duration of treatment through each therapy line is shown for all three cohorts in Fig. 1; see Box for corresponding German guidelines for the treatment of patients with RRMM.

\section{Patient and Disease Characteristics}

Over half $(60 \%)$ of patients were male, with a median age at initiation of current line of treatment of 72,72 , and 74 years for patients in second, third, and fourth or later line, respectively. Most patients were unemployed or retired at diagnosis, $80 \%, 82 \%$, and $87 \%$ at second-, third-, or fourth- or later-line treatment, respectively (Table 2). Overall, irrespective of treatment line, the majority of patients (more than $80 \%$ ) were either ISS stage 2 or 3 at diagnosis. Transplant history was similar across all treatment lines. In the second-line setting, patients who had received a prior transplant were more likely to receive daratumumab-based regimens than those who had not received a transplant in earlier lines $(53 \%$ vs. $35 \%$, respectively; $p=0.013$ ). However, there was a trend towards patients who had not received a prior transplant being more likely to receive second-line carfilzomib-based therapy compared with those who had undergone a transplant procedure ( $44 \%$ vs. $31 \%$, respectively; 


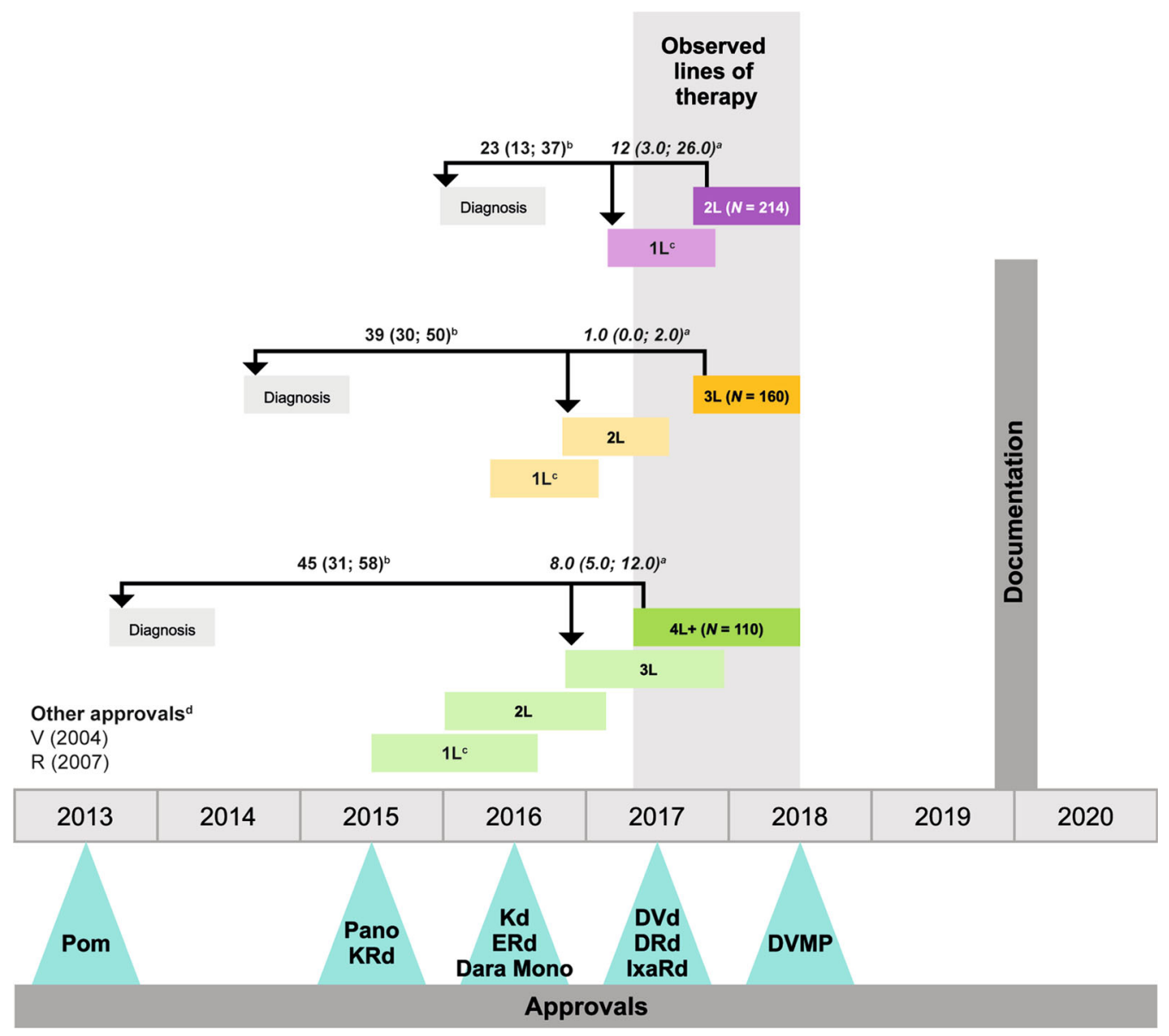

Fig. 1 Treatment pathway for patients receiving differing lines of therapy on entry into the study. Additional data relating to time between treatments for the main therapies and regimens is provided in Table S8. For $2 \mathrm{~L}$ and $3 \mathrm{~L}$, treatment received between October 2017 and June 2018; for $4 \mathrm{~L}+$, treatment received between May 2017 and June 2018. ${ }^{a}$ Median time to next treatment line in months (IQR). ${ }^{\mathrm{b}}$ Median time between diagnosis and initiation of current $M M$ treatment in months (IQR). ${ }^{c} 1 \mathrm{~L}$ maintenance treatment not included; induction treatment only. ${ }^{\mathrm{d} E C}$ approval date/launch date in Germany. $I L$ first line, $2 L$ second line, $3 L$ third line, $4 L+$ fourth or later line,

$p=0.071)$. In the third-line setting, use of daratumumab-based therapy was similar in patients with and without a transplant (48\% vs. $52 \%$, respectively; $p=0.655$ ), while carfilzomibbased therapy was used in $37 \%$ vs. $35 \%$ of patients with and without a transplant, respectively $(p=0.002)$. At fourth or later lines of treatment, use of carfilzomib-based regimens
Dara Mono daratumumab monotherapy, DRd daratumumab-lenalidomide-dexamethasone, $D V d$ daratumumab-bortezomib-dexamethasone, DVMP daratumumab-bortezomib-melphalan-prednisone, $E C$ European Commission, ERd elotuzumab-lenalidomide-dexamethasone, IQR interquartile range, IxaRd ixazomib-lenalidomide-dexamethasone, $K d$ carfilzomib-dexamethasone, $K R d$ carfilzomib-lenalidomide-dexamethasone, $M M$ multiple myeloma, Pano panobinostat, Pom pomalidomide, $R$ lenalidomide, $V$ bortezomib

was similar in patients who had received a transplant (35\%) compared with those who had not $(22 \% ; p=0.130)$. In contrast, daratumumab-based therapies were used more often in patients who had not received a transplant $(58 \%)$ than in those who had received a transplant $(35 \%, p=0.032)$. With respect to other regimens, the group sizes for transplant patients 


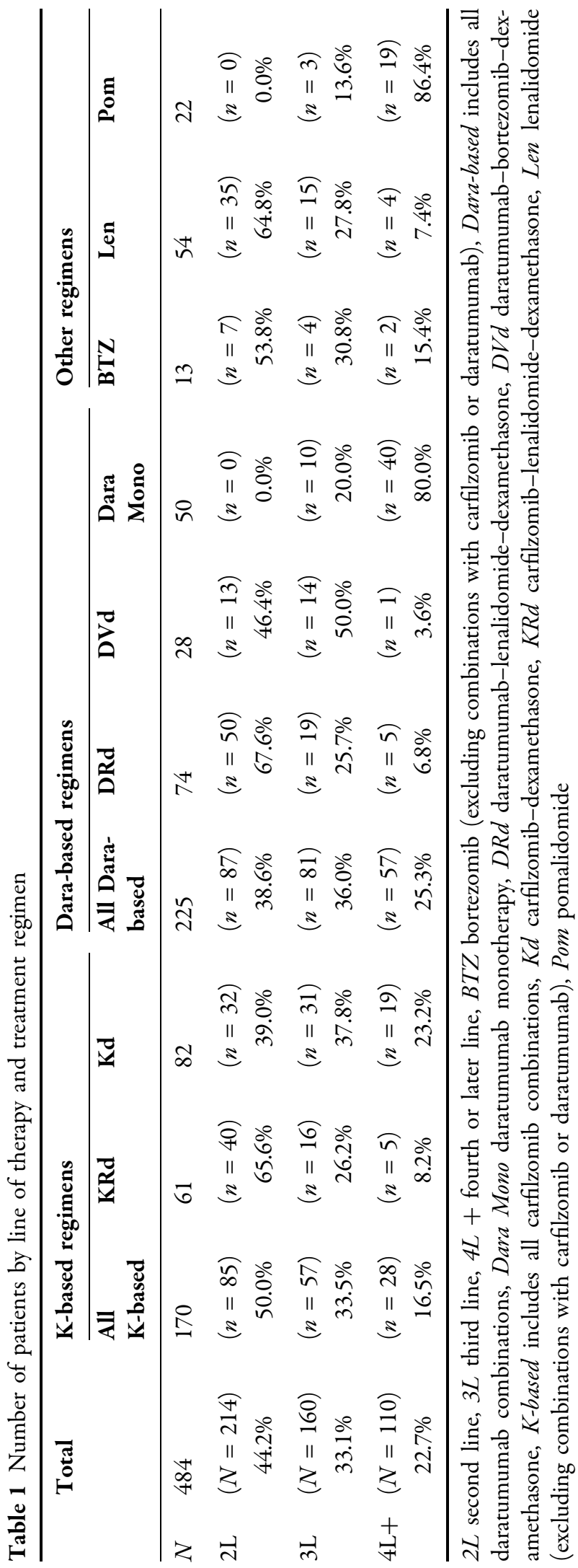

were all less than 10 and therefore no comparisons with non-transplant patients are reported here.

At diagnosis, bone pain and anemia were the most common symptoms, reported in $54 \%$ and $50 \%$ of all patients, respectively. Overall, almost one-third (30\%) of patients had hypercalcemia, while the frequency of renal dysfunction at diagnosis was $24 \%$ (Table 2). The median time between diagnosis and current line initiation was approximately 23 months for patients on second-line therapy, 39 months in third line, and 45 months in fourth- or later-line patients.

Among the second-line cohort $(N=214)$, the majority (73\%) of patients had an Eastern Cooperative Oncology Group Performance Status (ECOG PS) of $0-1$, with $23 \%$ having an ECOG PS of 2 at initiation of treatment (Table 2). Prior to initiation, approximately one-third $(32 \%)$ of patients had received a transplant and almost two-thirds of patients (63\%) were receiving osteoprotective drugs, predominantly zoledronate $(44 \%)$ and denosumab (42\%) (Table 2). Cytogenetic abnormalities were tested in almost three-quarters (73\%) of patients and revealed a prevalence of chromosome 13 deletion (del[13q]) in approximately $30 \%$, and $17 \mathrm{p} 13$ deletion in $17 \%$ of patients (Table 2).

The third-line cohort comprised 160 patients. Over half (58\%) of the patients had an ECOG PS of $0-1,34 \%$ had an ECOG PS of 2 at initiation of treatment, and $54 \%$ had received an ASCT (Table 2). Cytogenetic abnormalities were found in $71 \%$ of third-line patients tested, with $32 \%$ having del(13q) and $12 \%$ with $17 \mathrm{p} 13$ deletions. Approximately $70 \%$ of patients were receiving osteoprotective drugs at initiation of third-line therapy, mainly denosumab (51\%) and zoledronate (36\%).

In the fourth- or later-line cohort $(N=110)$, a high proportion (57\%) of patients had an ECOG PS of 2 at initiation (Table 2). Almost three-quarters of patients (70\%) were tested for cytogenetic abnormalities, $32 \%$ of whom had $\operatorname{del}(13 q)$ and a further $16 \%$ with $17 \mathrm{p} 13$ deletion. Nearly one-third (28\%) of patients underwent transplants and two-thirds (66\%) of patients received osteoprotective drugs at initiation 
Table 2 Characteristics of patients with RRMM following first-line relapse

\begin{tabular}{|c|c|c|c|}
\hline & $2 \mathbf{L}$ & $3 \mathbf{L}$ & $4 \mathrm{~L}+$ \\
\hline$N$ & 214 & 160 & 110 \\
\hline Male (\%) & 60.3 & 59.4 & 60.9 \\
\hline Age at diagnosis, median (IQR) years & $67(59-74)$ & $66(57-72)$ & $67(60-75)$ \\
\hline Age at initiation of current line, median (IQR) years & $72(64-78)$ & $72(65-78)$ & $74(67-81)$ \\
\hline $\begin{array}{l}\text { Time between diagnosis and initiation of current MM treatment, median } \\
\text { (IQR) months }\end{array}$ & $23(13-37)$ & $39(30-50)$ & $45(31-58)$ \\
\hline Treatment duration, median (IQR) months & $8(5-13)$ & $10(5-13)$ & $8(6-12)$ \\
\hline Ongoing treatment at time of data collection (\%) & 50.0 & 26.3 & 11.8 \\
\hline \multicolumn{4}{|l|}{ ECOG PS at initiation (\%) } \\
\hline $0-1$ & 73.4 & 58.1 & 36.4 \\
\hline 2 & 22.9 & 33.8 & 57.3 \\
\hline $3-4$ & 3.7 & 8.1 & 6.4 \\
\hline \multicolumn{4}{|l|}{ ISS stage at diagnosis (\%) } \\
\hline 1 & 8.9 & 16.8 & 13.7 \\
\hline 2 & 29.7 & 30.7 & 30.4 \\
\hline 3 & 61.5 & 52.6 & 55.9 \\
\hline Missing & 10.3 & 14.4 & 7.3 \\
\hline \multicolumn{4}{|l|}{ Symptoms at diagnosis (\%) } \\
\hline Bone pain & 54.7 & 56.6 & 48.2 \\
\hline Anemia & 46.3 & 48.4 & 60.0 \\
\hline Hypercalcemia & 28.0 & 29.6 & 26.4 \\
\hline Renal dysfunction & 24.8 & 26.4 & 20.9 \\
\hline \multicolumn{4}{|l|}{ Cytogenetic abnormalities (\%) } \\
\hline$n^{\mathrm{a}}$ & 157 & 114 & 76 \\
\hline $\operatorname{del}(17 \mathrm{p} 13)$ & 16.7 & 12.3 & 15.8 \\
\hline $\mathrm{t}(4 ; 14)$ & 2.5 & 10.5 & 14.5 \\
\hline $\mathrm{t}(14 ; 16)$ & - & 7.9 & 3.9 \\
\hline $\operatorname{del}(13 q)$ & 30.6 & 31.6 & 31.6 \\
\hline $\operatorname{amp}(1 \mathrm{q} 21)$ & 15.3 & 14.0 & 18.4 \\
\hline $\mathrm{t}(11 ; 14)$ & 7.6 & 5.3 & 10.5 \\
\hline \multicolumn{4}{|l|}{ Transplant history (\%) } \\
\hline$n^{\mathrm{b}}$ & 68 & 54 & 31 \\
\hline Prior ASCT & 31.8 & 33.8 & 28.2 \\
\hline
\end{tabular}


Table 2 continued

\begin{tabular}{llll}
\hline & 2L & 3L & 4L+ \\
\hline Single transplant & 60.3 & 57.4 & 54.8 \\
Transplant before 2L & 95.0 & 42.6 & 54.8 \\
Transplant during 2L & 5.0 & 8.7 & 0.0 \\
Use of osteoprotective drugs at diagnosis (\%) & 62.6 & 60.0 & 51.8 \\
Use of osteoprotective drugs at initiation of current line (\%) & 69.6 & 69.4 & 65.5 \\
$n^{\text {c }}$ & 149 & 111 & 57 \\
Zoledronate (\%) & 43.6 & 36.0 & 64.9 \\
Denosumab (\%) & 41.6 & 50.5 & 17.5 \\
Pamidronate (\%) & 8.1 & 6.3 & 5.3 \\
Ibandronate (\%) & 6.7 & 7.2 & 12.3 \\
LDH at initiation $(\mathrm{U} / \mathrm{L})$, median (IQR) & 236 & 236 & 246 \\
& $(188-298)$ & $(196-310)$ & $(19-320)$ \\
$n^{\mathrm{d}}$ & 189 & 146 & 101 \\
Proportion with $\mathrm{LDH}>240 \mathrm{U} / \mathrm{L}(\%)^{\text {Proportion with } \mathrm{Hb}<10 \mathrm{~g} / \mathrm{dL}(\%)^{\mathrm{e}}}$ & 47.6 & 46.6 & 54.6 \\
\hline
\end{tabular}

$2 L$ second line, $3 L$ third line, $4 L+$ fourth or later line, $A S C T$ autologous stem cell transplant, ECOG PS Eastern Cooperative Oncology Group performance status, $H b$ hemoglobin, IQR interquartile range, ISS International Staging System, $L D H$ lactic dehydrogenase, $M M$ multiple myeloma, $R R M M$ relapsed and/or refractory multiple myeloma

${ }^{a}$ Number of patients tested in each line for cytogenetic abnormalities

${ }^{b}$ Number of patients in each line with at least one prior transplant

${ }^{c}$ Number of patients using osteoprotective drugs in respective lines

${ }^{\mathrm{d}}$ Number of patients in each line with available LDH level

${ }^{e}$ Patients in each line with available Hb level; $n=209, n=159$, and $n=109$ for $2 \mathrm{~L}$, 3L, and 4L+ respectively

(Table 2), mainly zoledronate (65\%) and denosumab (18\%).

Similar median lactate dehydrogenase (LDH) levels were observed across all treatment lines (second line, $236 \mathrm{U} / \mathrm{L}$; third line, $236 \mathrm{U} / \mathrm{L}$; and fourth or later line, $246 \mathrm{U} / \mathrm{L}$ ), while the proportions of patients with $\mathrm{LDH}>240 \mathrm{U} / \mathrm{L}$, which is associated with a poorer prognosis, were similar across second- and third-line treatment groups (48\% and $47 \%$, respectively) and was $55 \%$ in fourth and later lines of treatment (Table 2). The proportion of patients with hemoglobin levels $<10 \mathrm{~g} / \mathrm{dL}$, also conferring higher risk, increased across treatment lines (second line, 41\%; third line, 48\% and fourth and later lines, 56\%).

\section{Treatment Patterns and Patient Profile by Treatment Regimen}

Individual treatments, monotherapy, and combinations for each therapy line are shown in Table S1 in the electronic supplementary material. After failure to respond to first-line treatment, most patients received second-line treatment with daratumumab- or carfilzomibbased therapies $(41 \%$ and $40 \%$, respectively; Fig. 2, Table S1); $20 \%$ of patients were given other treatments (lenalidomide- or bortezomib- 
based therapies; Fig. 2, Table S2). For 107 patients (50\%) who had completed second-line therapy at the time of data collection, the median (IQR) treatment duration was 8 (5-13) months; 9 (5-13) months for carfilzomib patients, 8 (7-13) months for daratumumab patients, and 6 (5-14) months for lenalidomide patients (Table S2). Daratumumab-based treatment was prescribed to $61 \%$ of patients at ISS stage 3 and $41 \%$ of those with a prior ASCT (Fig. 3, Table S2).

In patients receiving third-line therapy, daratumumab-based regimens were used in 51\% of patients, most frequently in symptomatic patients, while carfilzomib- and lenalidomidebased regimens were used in 36\% and $9 \%$ of patients, respectively (Fig. 2, Table S3). In thirdline patients with prior ASCT, a similar proportion of patients were treated with daratumumab- or carfilzomib-based treatment

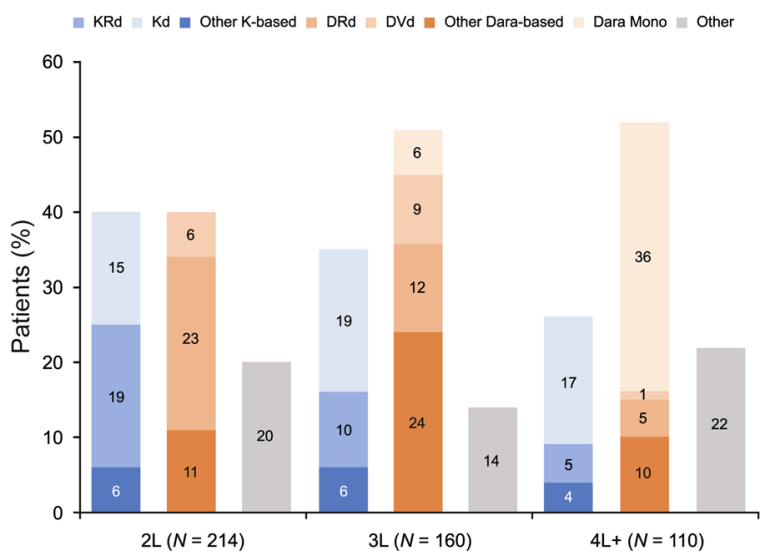

Fig. 2 Patterns of treatment-based regimens by line of therapy at time of data abstraction. Data were abstracted between 10 Oct 2019 and 22 Feb 2020. $2 L$ second line, $3 L$ third line, $4 L+$ fourth or later line, Dara Mono daratumumab monotherapy, DRd daratumumab-lenalidomide-dexamethasone, $D V d$ daratumumab-bortezomibdexamethasone, $K d$ carfilzomib-dexamethasone, KRd carfilzomib-lenalidomide-dexamethasone, Other Darabased includes all daratumumab combinations excluding DRd, DVd, or Dara Mono, Other K-based includes all carfilzomib combinations excluding KRd or $\mathrm{Kd}$, Other all other regimens that do not include carfilzomib or daratumumab regimens, 32\% and 35\%, respectively (Table S3). Bortezomib-based regimens were only used in $3 \%$ of patients in third line, mostly in combination with other agents. Almost 20\% of patients in third line received Kd and $12 \%$ DRd. For 118 patients who had completed third line at the time of data collection, the median (IQR) treatment duration was 10 (5-13) months; 10 (5-13) months for carfilzomib patients, 10 (7-13) months for daratumumab patients, and 7 (5-13) months for lenalidomide patients (Fig. 3, Table S3).

Most patients on fourth- or later-line therapy received daratumumab-based regimens (52\%) and approximately $25 \%$ of patients received carfilzomib-based regimens. Daratumumab was used as monotherapy in 36\% of patients (Fig. 2, Table S4). Lenalidomide was rarely used in fourth or later line (4\%), while pomalidomide was used in $16 \%$ of patients. For fourth- or laterline patients with prior ASCT numbers were small; $44 \%$ were treated with carfilzomib-based regimens and 39\% received pomalidomide regimens (Table S4). For patients who completed fourth or later line at the time of data

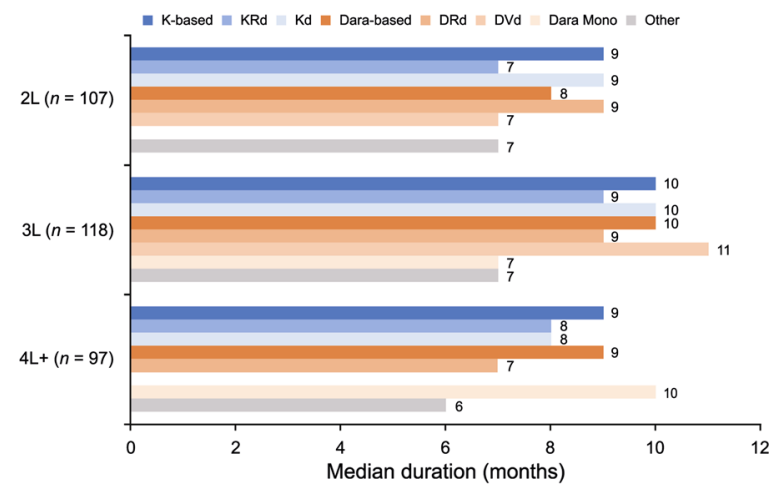

Fig. 3 Duration of treatment by regimen and line of therapy. $2 L$ second line, $3 L$ third line, $4 L+$ fourth or later line, Dara-based includes all daratumumab combinations, Dara Mono daratumumab monotherapy, DRd daratumumab-lenalidomide-dexamethasone, $D V d$ daratumumab-bortezomib-dexamethasone, $K$-based includes all carfilzomib-based combinations, $K d$ carfilzomib-dexamethasone, KRd carfilzomib-lenalidomide-dexamethasone, Other all other regimens that do not include carfilzomib or daratumumab 
Table 3 Response (\%) assessed in patients with RRMM by therapy line and treatment regimen

\begin{tabular}{|c|c|c|c|c|c|c|c|c|c|c|c|}
\hline & Total & $\begin{array}{l}\text { K- } \\
\text { based }\end{array}$ & KRd & Kd & $\begin{array}{l}\text { Dara- } \\
\text { based }\end{array}$ & DRd & DVd & $\begin{array}{l}\text { Dara } \\
\text { Mono }\end{array}$ & $\begin{array}{l}\text { Other } \\
\text { BTZ }\end{array}$ & $\begin{array}{l}\text { Other } \\
\text { Len }\end{array}$ & $\begin{array}{l}\text { Pom- } \\
\text { based }\end{array}$ \\
\hline $2 \mathrm{~L}(n)$ & 191 & 77 & 39 & 30 & 73 & 44 & 12 & 0 & 7 & 34 & 0 \\
\hline ORR & 90.6 & 89.7 & 89.7 & 86.7 & 94.5 & 93.1 & 100.0 & 0.0 & 71.4 & 85.8 & 0.0 \\
\hline CR & 22.0 & 16.9 & 15.4 & 16.7 & 38.4 & 40.9 & 25.0 & 0.0 & 0.0 & 2.9 & 0.0 \\
\hline VGPR & 42.4 & 45.5 & 48.7 & 36.7 & 34.2 & 38.6 & 25.0 & 0.0 & 57.1 & 50.0 & 0.0 \\
\hline PR & 26.2 & 27.3 & 25.6 & 33.3 & 21.9 & 13.6 & 50.0 & 0.0 & 14.3 & 35.3 & 0.0 \\
\hline SD & 5.2 & 5.2 & 5.1 & 6.7 & 1.4 & 2.3 & 0.0 & 0.0 & 28.6 & 8.8 & 0.0 \\
\hline $\mathrm{PD}$ & 4.2 & 5.2 & 5.1 & 6.7 & 4.1 & 4.5 & 0.0 & 0.0 & 0.0 & 2.9 & 0.0 \\
\hline $3 \mathrm{~L}(n)$ & 139 & 49 & 15 & 25 & 72 & 17 & 13 & 9 & 4 & 12 & 2 \\
\hline ORR & 84.2 & 85.7 & 86.7 & 84 & 87.4 & 76.5 & 92.4 & 77.7 & 100.0 & 66.6 & 0.0 \\
\hline CR & 25.9 & 16.3 & 0.0 & 16.0 & 31.9 & 29.4 & 38.5 & 0.0 & 25.0 & 33.3 & 0.0 \\
\hline VGPR & 28.8 & 24.5 & 60.0 & 12.0 & 31.9 & 41.2 & 15.4 & 33.3 & 50.0 & 25.0 & 0.0 \\
\hline PR & 29.5 & 44.9 & 26.7 & 56.0 & 23.6 & 5.9 & 38.5 & 44.4 & 25.0 & 8.3 & 0.0 \\
\hline SD & 10.1 & 10.2 & 6.7 & 12.0 & 5.6 & 11.8 & 7.7 & 0.0 & 0.0 & 33.3 & 50.0 \\
\hline PD & 5.8 & 4.1 & 6.7 & 4.0 & 6.9 & 11.8 & 0.0 & 22.2 & 0.0 & 0.0 & 50.0 \\
\hline $4 \mathrm{~L}+(n)$ & 106 & 28 & 5 & 19 & 55 & 5 & 1 & 39 & 2 & 3 & 17 \\
\hline ORR & 61.3 & 71.4 & 80.0 & 73.7 & 63.6 & 20.0 & 100.0 & 69.2 & 100.0 & 33.3 & 41.1 \\
\hline CR & 15.1 & 14.3 & 0.0 & 15.8 & 14.5 & 0.0 & 0.0 & 20.5 & 0.0 & 33.3 & 17.6 \\
\hline VGPR & 23.6 & 21.4 & 40.0 & 21.1 & 30.9 & 0.0 & 100.0 & 35.9 & 50.0 & 0.0 & 5.9 \\
\hline PR & 22.6 & 35.7 & 40.0 & 36.8 & 18.2 & 20.0 & 0.0 & 12.8 & 50.0 & 0.0 & 17.6 \\
\hline SD & 24.5 & 25.0 & 20.0 & 21.1 & 27.3 & 40.0 & 0.0 & 25.6 & 0.0 & 66.7 & 5.9 \\
\hline PD & 14.2 & 3.6 & 0.0 & 5.3 & 9.1 & 40.0 & 0.0 & 5.1 & 0.0 & 0.0 & 52.9 \\
\hline
\end{tabular}

Data should be interpreted with caution where numbers of subjects treated with a specific regimen are small $2 L$ second line, $3 L$ third line, $4 L+$ fourth or later line, $B T Z$ bortezomib (excluding combinations with carfilzomib or daratumumab), $C R$ complete response, Dara-based includes all daratumumab combinations, Dara Mono daratumumab monotherapy, $D R d$ daratumumab-lenalidomide-dexamethasone, $D V d$ daratumumab-bortezomib-dexamethasone, $K$-based includes all carfilzomib combinations, $K d$ carfilzomib-dexamethasone, $K R d$ carfilzomib-lenalidomide-dexamethasone, Len lenalidomide (excluding combinations with carfilzomib or daratumumab), ORR overall response rate (defined as PR, VGPR, and CR or better), PD progressive disease, Pom pomalidomide, $P R$ partial response, $R R M M$ relapsed and/or refractory multiple myeloma, $S D$ stable disease, $V G P R$ very good partial response

extraction, the median (IQR) treatment duration was 8 (6-12) months; 9 (6-12) months for carfilzomib, 9 (7-13) months for daratumumab,
6 (2-6) months for lenalidomide, and 7 (5-11) months for pomalidomide patients (Fig. 3, Table S4). 


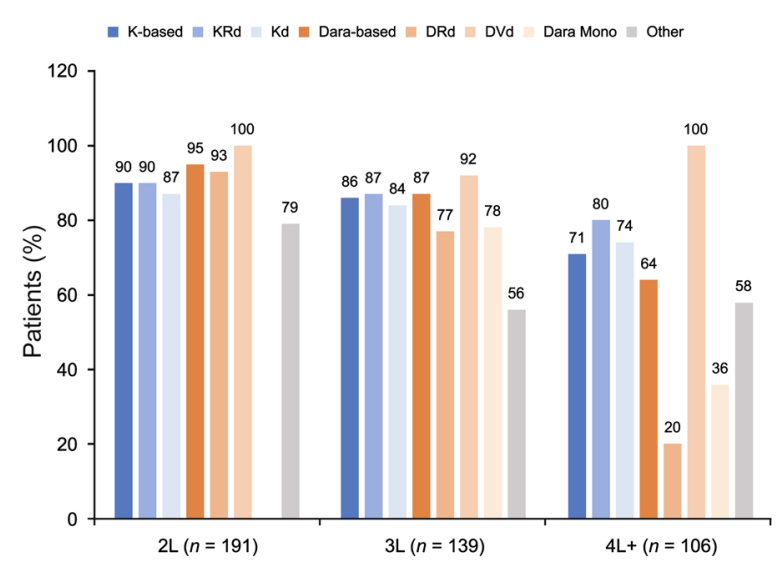

Fig. 4 Overall response rates in patients with RRMM by line of therapy. $2 L$ second line, $3 L$ third line, $4 L+$ fourth or later line, Dara-based includes all daratumumab combinations, Dara Mono daratumumab monotherapy, $D R d$ daratumumab-lenalidomide-dexamethasone, $D V d$ daratumumab-bortezomib-dexamethasone, K-based includes all carfilzomib-based combinations, $K d$ carfilzomib-dexamethasone, KRd carfilzomib-lenalidomide-dexamethasone, Other all other regimens that do not include carfilzomib or daratumumab, $R R M M$ relapsed and/or refractory multiple myeloma

\section{Response Rates}

Where response was available for patients in second line $(n=191 / 214)$, the overall response rate (ORR; defined as partial response [PR], very good partial response [VGPR], and complete response [CR]) was $91 \%$, attributed to $95 \%$ in patients on daratumumab-based regimens, $90 \%$ in patients on carfilzomib-based regimens, and $86 \%$ in patients receiving other lenalidomidebased regimens (Table 3; Fig. 4). High CR rates were observed among patients who received DRd $(n=44)$ and DVd $(n=12)(41 \%$ and $25 \%$, respectively) in second line. CR rates for KRd $(n=39)$ and $\mathrm{Kd}(n=30)$ patients were $15 \%$ and $17 \%$, respectively (Table 3 ). Where response was available for patients in third line $(n=139)$, an OR was achieved by $84 \%$ of patients overall, including $87 \%$ of daratumumab patients and $86 \%$ of carfilzomib patients. CR rates of $29 \%$ and 39\% were observed for patients who received DRd $(n=17)$ and DVd $(n=13)$. CR rates were $0 \%$ and $16 \%$ for those who received
KRd $(n=15)$ and $\mathrm{Kd}(n=25)$, respectively (Table 3). For patients in fourth or later line, where response was available $(n=106), 61 \%$ of patients achieved an OR, including $64 \%$ of daratumumab patients, $71 \%$ of carfilzomib patients, and $41 \%$ of pomalidomide patients, although actual patient numbers were small (Table 3, Fig. 4). The response rates in a number of subgroups presented in Table 3 need to be interpreted with caution because of the small sample size.

Approximately $22 \%$ of patients had MRD tests performed at time of best response during their second-line therapy, most via flow cytometry-based assays. MRD negativity was recorded in $15 \%$ of tested patients. For patients in third line, of $24 \%$ of tests conducted, $18 \%$ reported MRD negativity, while in fourth- or later-line patients, $10 \%$ of tests conducted were negative for MRD.

\section{Hospitalizations}

Of the 214 patients in current second-line therapy, 35 patients (16\%) had at least one unplanned in-patient hospitalization day related to treatment administration (Table 4). The mean number of in-patient hospitalizations (longer than 1 day), excluding the 1-day hospitalization related to treatment administration, was 2.8 (Table 4). The mean number of days spent in in-patient hospitalizations related to treatment administration was 14.4 days, and 10.0 days for hospitalizations related to treatment toxicity (Table 4$)$. A total of $25(16 \%)$ patients in third line also had unplanned hospitalizations, with a mean hospital stay of 17.7 days related to treatment administration and 9.6 days due to treatment toxicity (Table 4). Few patients were reported in fourth or later lines, with $11 \%(12 / 110)$ reporting a mean of 5.2 unplanned hospitalizations, with a mean total of 17.9 days related to treatment administration and 11.7 days due to treatment toxicity (Table 4). In fourth or later lines, carfilzomib patients required fewer hospitalization days to receive treatment compared with earlier lines, while daratumumab patients had twice as many hospitalization days to receive their treatment 


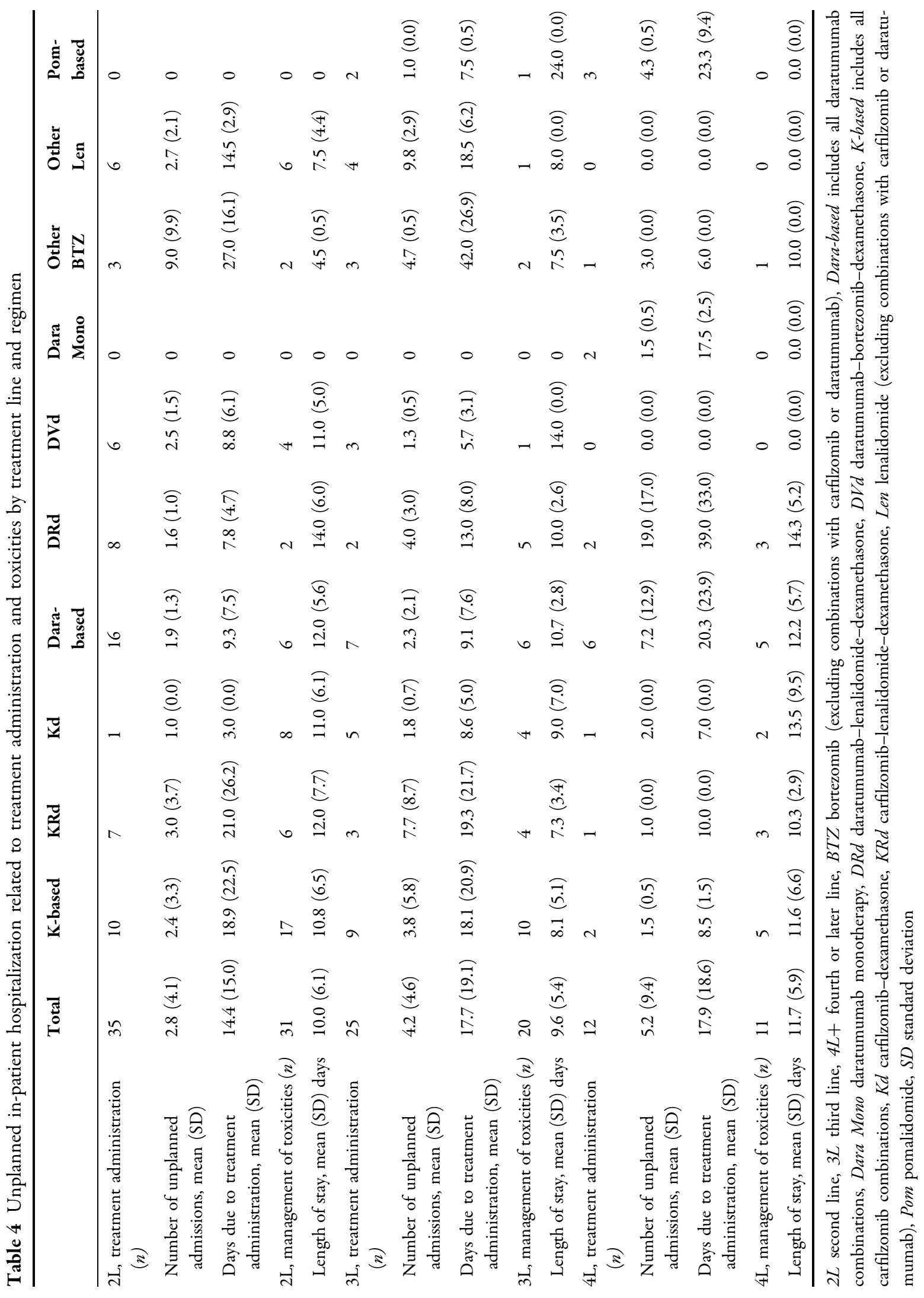


and had also more associated hospitalizations due to treatment toxicity than in earlier lines (Table 4).

\section{DISCUSSION}

This study is supplemental to our previous analysis which focused on the use of proteasome inhibitors in patients with RRMM in Germany $[12,19]$ and now examines the realworld management of RRMM with novel agents. Data were collected from case report forms completed by hematologists, oncologists, or hemato-oncologists engaged in the treatment of RRMM in Germany between October 2019 and February 2020, providing an insight into the therapeutic strategies in different lines of therapy in routine clinical practice reflective of the current label between May 2017 and June 2018. The results of this analysis provide valuable information about the clinical profile of patients which, in turn, plays a vital role in the choice of the treatment of multiple myeloma.

In this study, baseline patient characteristics at diagnosis were generally similar across patients in all lines of therapy. The median age of patients at diagnosis was comparable to other reports [12, 20,21]. Despite the small number of patients, some minor differences could be noted regarding the age of patients among treatment groups. For example, the median age of patients at initiation of second-line treatment was slightly lower for those treated with daratumumab-based compared to carfilzomib-based regimens (70 vs. 72 years), significantly for KRd and DRd (67 vs. 70 years; $p<0.05$ ), but not for Kd and DVd (74 vs. 75 years) (Table S2). As has been previously described in RRMM [5], the median treatment-free interval was shorter between third- and fourth-line treatment than it was between first- and second-line treatment in this study. Interestingly, the shortest treatment-free interval was observed between second- and third-line treatment. This was somewhat unexpected but may be related to the fact that there has been a change in how patients with RRMM are managed-they now receive treatment until progression rather than a standard six cycles of treatment followed by a treatment holiday. In line with this, the reason for discontinuing second- and third-line treatment was progression for $47 \%$ and $62 \%$ of patients, respectively. Nonetheless, the median overall times from first- to second-line therapy (12 months) and from first- to third-line therapy (29 months) were more in line with expectations when the median duration of secondline therapy (8 months) was also considered. However, it should also be remembered that this was a descriptive cross-sectional survey and that the observed timings and durations of treatment would need to be confirmed in a longitudinal survey study.

The ISS score 3 is associated with a worse prognosis and lower median survival than ISS scores 1 and 2 (29 months vs. 62 months and 44 months, respectively) [6]. In this analysis, the proportion of patients across all lines of therapy with ISS score 3 at diagnosis was higher $(57 \%)$ than that reported previously (24-49\%) $[16,21-23]$. Only $13 \%$ of patients had ISS score 1 , a similar proportion to that described by Merz et al. (8-15\%), but lower than in other analyses (16-24\%) [16, 21, 23]. Despite the large proportion of high-risk patients, the percentage of patients in remission was slightly higher $(75-78 \%)$ than that reported in other analyses (66-73\%) [16, 22, 23].

For patients in second and third lines, the proportions of patients initiating their therapies with ECOG PS $\geq 2$ were similar to those reported by Despiégel et al. (27\% vs. $21 \%$ and $20 \%$, and $42 \%$ vs. $45 \%$ and $36 \%$, respectively) $[15,24]$ but lower than those reported by Merz et al. (43\% and 50\%, respectively) [25]. In this analysis, the proportion of patients with ECOG PS 0-1 decreased in later lines of therapy: almost $80 \%(78 \%)$ of fourth-line patients had ECOG PS $0-1$ at diagnosis compared with only $36 \%$ at fourth-line initiation. The proportion of patients in fourth-line initiation with ECOG PS 0-1 was lower than that reported by Despiégel et al. and Engelhardt et al. (36\% vs. $57 \%$ and $54 \%$, respectively) $[15,24]$.

At diagnosis, most patients fulfilled the CRAB (calcium elevation, renal dysfunction, anemia, bone disease) criteria, with bone pain and anemia being the most common symptoms, present in $54 \%$ and $50 \%$ of all patients, 
respectively. Anemia (hemoglobin level $<10 \mathrm{~g} / \mathrm{dL}$ ) was more frequent in patients on later lines of therapy. Up to two-thirds of patients received zoledronate, an osteoprotective drug, at the start of treatment, while the usage of denosumab increased up to $50 \%$ in third line, consistent with approval in March 2018 and recommendations [26, 27]. In addition, approximately one-third of patients (30\%) had hypercalcemia, and around one-quarter of patients $(24 \%)$ had renal dysfunction at diagnosis. All of these symptoms were slightly higher than our previous study [12] but similar to other reports [16, 22, 28, 29] and highlight their value in aiding decisions on the treatment of patients who are at high risk of disease progression and who would benefit from therapy [30].

Overall, 30\% of patients of all lines of therapy had at least one ASCT, and most patients received ASCT before second line, comparable to that reported by Raab et al. (31\%) [21]. In the analysis of second-line treatment regimens in the present study, substantially more patients with prior transplantation were likely to receive daratumumab-based regimens (mainly DRd) than carfilzomib-based regimens and this differs from a previous study where KRd was the most common regimen in previously transplanted German patients [12]. In second-line transplant-ineligible patients, DRd use was approximately half that seen in transplant-eligible patients and KRd use was very similar irrespective of patient transplant status. Around $70 \%$ of patients underwent cytogenetic testing; the prevalence of del(13q), a marker of poor prognosis, was approximately $30 \%$, with $\mathrm{t}(14 ; 16)$, $\mathrm{t}(4 ; 14)$, and amp(1q21) prevalence of $4-8 \%$, $3-15 \%$, and $14-18 \%$, respectively. $17 \mathrm{p} 13$ deletion was reported in about $12-17 \%$ of all patients, compared with $8-10 \%$ reported previously [31]. These results showed a higher frequency of cytogenetic testing than previously reported (ranging from 69\% to 73\%) [20] while, despite the absence of cytogenetic-risk assessment guidelines, cytogenetic testing in Germany has been reported to have increased from $23 \%$ in 2008 to $35 \%$ in 2009 and 53\% in 2011 $[32,33]$.
The regimens most frequently prescribed were DRd in second line, Kd in third line, and daratumumab monotherapy in fourth or later line. Patterns of treatment differed significantly across subsequent lines but were broadly similar to other studies in Germany [34, 35], including one focusing on proteasome inhibitor use $[12,19]$.

The benefit of adding a monoclonal antibody to $\mathrm{Kd}$ or $\mathrm{Vd}$ has been demonstrated in several phase 3 trials [5]. In our study, regimens with second-generation proteasome inhibitors and monoclonal antibodies, including carfilzomib and daratumumab, were mostly used in advanced lines of treatment, while lenalidomide was less frequently prescribed in combinations without carfilzomib and/or daratumumab. Similarly, lenalidomide was rarely prescribed in fourth-line therapy. In part, this could be explained by the introduction of pomalidomide [36], although this drug was rarely used when compared with carfilzomiband daratumumab-containing regimens, possibly because of restricted reimbursement.

Interestingly, favorable response rates were maintained across all treatment lines, with similar CR achieved in fourth line with both carfilzomib- and daratumumab-based regimens (14\% and $15 \%$ ) and ORRs of $71 \%$ and $64 \%$, respectively. MRD was assessed in almost onequarter of the patients in clinical practice, despite the lack of requirement to test in realworld practice, and this may reflect the interest in using this outcome, although it is not recognized as an efficacy endpoint by regulatory authorities. There are, however, limitations in interpreting the small proportion of patients achieving MRD negativity as the response status among patients tested for MRD was not documented. Lastly, the requirement for hospitalization was similar across all treatment lines, with longer hospital stays due to treatment administration than for treatment toxicity.

The results of the present study differ from earlier treatment pattern data reported for Europe and elsewhere [20-22]. For example, an analysis of real-world practice patterns in seven countries across Europe in 2014 showed that lenalidomide-based regimens were used most frequently in the second-line setting, following 
first-line bortezomib-based therapy [21]. Similar treatment patterns were seen in a prospective registry study including data for patients treated in 22 countries across Europe, the Middle East, and Africa between 2010 and 2014 [20]. An updated analysis of real-world treatment patterns in Europe from Raab et al., comparing data from 2014 with that in 2016, showed an increasing use of second-generation agents and monoclonal antibodies in the second-line setting and a decreasing use of third-line lenalidomide-based regimens (other than in combination with novel agents) [36], which is more in line with observations in the current study. They also found that fourth-line regimens were more diverse than were observed in 2014 [36]. The present data also contrast with findings from a real-world US study of patients initiating first-line multiple myeloma treatment between 2011 and 2017, in which bortezomib and lenalidomide were the most commonly used agents in both the first- and second-line settings [37]. In that study, carfilzomib was the most frequently used third-line agent, but daratumumab was used infrequently across treatment lines at the time of that study [37]. Although in both Europe and the USA bortezomib is the most commonly used proteasome inhibitor in the first-line setting, and in combination with novel agents in second and later lines [36, 37], in our study, it was prescribed mainly in combination with daratumumab in second and third lines (9\% and 11\%, respectively). Overall, there are clear differences in treatment practice between countries [20, 21, 37-39], with no standard of care for patients with RRMM in later lines of treatment, and treatment algorithms evolving over time $[21,36,37,39]$. The changing landscape and treatment practice differences between countries are related to the introduction of various novel therapies over recent years, as the timings of regulatory approvals and reimbursement differ between Europe and the USA, and the time taken for such agents to become available after approval varies across Europe [36]. Of note, in Germany, novel treatments are available/reimbursed immediately after regulatory approval, meaning that these agents are often available earlier in Germany than elsewhere
$[36,39]$. Data from the present German study, therefore, provide useful insights into the uptake of novel agents in this rapidly changing treatment landscape.

A limitation of this non-interventional study is that the inclusion of data on patients with RRMM was not controlled, allowing larger centers to include more patients based on the size of the eligible population at each site. Despite the limitations that an unevenly distributed sample across participating sites may have, this population was considered to be generally representative of patients with RRMM treated in second to fourth and later lines in Germany, although most participating centers were from the state of Nordrhein-Westfalia. Notably, in 2008 there were 1717 medical oncologists registered in Germany as a whole and this was predicted to increase to 3806 in 2020 [40]. This means that the 515 physicians who were contacted to participate in the current study represented approximately $14 \%$ of all medical oncologists in Germany in 2020. Considering the stringent criteria that were included to ensure recruitment of physicians actively involved in the management of patients with multiple myeloma, the final sample should give a useful insight into clinical practice in Germany over the study period. Furthermore, patients included in the study were selected consecutively, in chronological order rather than as a random selection over the defined time period. As a result of differences in clinical practice and the reimbursement status of novel therapies between countries, these findings are not likely to be generalizable to countries outside of Germany, although it may provide early insights into future treatment patterns in other countries as and when novel agents become available. Furthermore, as this was a retrospective chart review, relapse was defined by physicians according to routine clinical practice and so would have been assessed in a variety of different ways, e.g., based on biochemical relapse, as indicated by rapidly increasing paraprotein levels, or the development of new/worsening of CRAB symptoms. No comparison was made between the different treatment combinations, because of the descriptive nature of the study, heterogeneity of the 
different treatment cohorts which cannot be considered as treatment sequences, dates of approval, and limited sample size. Overall, the retrospective nature of the study design and the patient information, such as out of pocket expenses or treatment preferences, limits the outcomes and interpretation.

\section{CONCLUSIONS}

The study reflects real-world clinical practice and provides an insight into the daily management of RRMM over differing lines of therapy and treatment regimens across Germany between May 2017 and June 2018. Although patterns of treatment regimens differ by line of therapy and are adapted as disease progresses and ECOG PS increases, patients mostly receive combination regimens with carfilzomib or daratumumab in second and third lines, with lenalidomide often combined with novel agents in second line. Regardless of the treatment choice, especially for high-risk patients, high ORRs were achieved in all lines.

\section{ACKNOWLEDGEMENTS}

Funding. This study was funded by Amgen; Amgen also paid the journal's Rapid Service and Open Access fees. Study conduction and statistical analysis were performed by Kantar Health.

Medical Writing, Editorial, and Other Assistance. Medical writing support was provided by Carine Thual of Amgen (Europe) $\mathrm{GmbH}$ and K. Ian Johnson, Bioscript Medical Ltd, funded by Amgen. The authors would like to acknowledge all centers that participated in this study.

Prior Presentation. This manuscript is based on work that has been previously presented as a poster at the annual meeting of the American Society of Hematology in 2020 and is published in Blood 2020;136 (S1):32. Available from https://doi.org/10.1182/blood-2020-136491.
Authorship. All named authors meet the International Committee of Medical Journal Editors (ICMJE) criteria for authorship for this article, take responsibility for the integrity of the work as a whole, and have given their approval for this version to be published.

Author Contributions. H. Tilman Steinmetz, Moushmi Singh, Joseph Milce, Mohamad Haidar, Achim Rieth, Andrea Lebioda, and Jörn Kohnke contributed to the study conception and design. Material preparation and data collection were performed by H. Tilman Steinmetz, Joseph Milce, Mohamad Haidar, and Jörn Kohnke. Data analysis was performed by Joseph Milce and Mohamad Haidar. H. Tilman Steinmetz, Moushmi Singh, Joseph Milce, Mohamad Haidar, Achim Rieth, Andrea Lebioda, and Jörn Kohnke commented on all previous drafts of the paper and read and approved the final manuscript.

Disclosures. H. T. Steinmetz has received research funding from Accord Healthcare, Amgen, Celgene, Novartis, Vifor; he has membership on an entity's board of directors, speaker's bureau, or its advisory committees with Alexion, Accord Healthcare, Amgen, Ariad, Bluebird Bio, BMS, Boehringer, Celgene, HexalSandoz, Novartis, Janssen-Cilag, Omnicare, Oncopeptides, Otsuka, Pfizer, Sanofi, Shire, TAD, Takeda, Vifor; and has received travel grants from Alexion, Amgen, Bayer, BMS, Boehringer, Celgene, Janssen-Cilag, Novartis, Omnicare, Sanofi, Takeda. M. Singh was an employee of Amgen at the time of the study and is currently an employee of Abbvie. J. Milce is an employee of Kantar Health who received funding to conduct this research. M. Haidar is an employee of Kantar Health who received funding to conduct this research. A. Rieth is an employee of Amgen and holds Amgen Stock. A. Lebioda is an employee of Amgen and holds Amgen Stock. J. Kohnke has lectured for Boehringer, Roche, Incyte, Novartis, Celgene, advisory committees with Amgen, Celgene, Pfizer, Novartis, Janssen-Cilag, Roche, Boehringer, Apogepha, and received travel grants from Roche, Boehringer, Celgene, GHD, Amgen, GSK, Janssen-Cilag. 
Compliance with Ethics Guidelines. This study was conducted retrospectively from data obtained for clinical purposes and complied with all relevant market research guidelines and legal obligations [19]. No identifiable protected health information was extracted during the study, and we consulted with the institutional review board (IRB) of Nordrhein (Düsseldorf) who determined that our study did not need ethical approval owing to the anonymization of the data collected. An official waiver of ethical approval was granted from the IRB of Nordrhein (Düsseldorf) on July 1, 2019.

Data Availability. The datasets generated during and/or analyzed during the current study are not publicly available. Amgen holds the source data and all authors had full access to the data. Qualified researchers may request data from Amgen clinical studies. Complete details are available at http://www.amgen.com/ datasharing.

Open Access. This article is licensed under a Creative Commons Attribution-NonCommercial 4.0 International License, which permits any non-commercial use, sharing, adaptation, distribution and reproduction in any medium or format, as long as you give appropriate credit to the original author(s) and the source, provide a link to the Creative Commons licence, and indicate if changes were made. The images or other third party material in this article are included in the article's Creative Commons licence, unless indicated otherwise in a credit line to the material. If material is not included in the article's Creative Commons licence and your intended use is not permitted by statutory regulation or exceeds the permitted use, you will need to obtain permission directly from the copyright holder. To view a copy of this licence, visit http://creativecommons.org/licenses/by$\mathrm{nc} / 4.0 /$.

\section{REFERENCES}

1. Bobin A, Liuu E, Moya N, et al. Multiple myeloma: an overview of the current and novel therapeutic approaches in 2020. Cancers (Basel). 2020;12(10): 2885. https://doi.org/10.3390/cancers12102885.

2. Ferlay J, Steliarova-Foucher E, Lortet-Tieulent J, et al. Cancer incidence and mortality patterns in Europe: estimates for 40 countries in 2012. Eur J Cancer. 2013;49(6):1374-403. https://doi.org/10. 1016/j.ejca.2012.12.027.

3. Robert Koch Institute. Cancer in Germany in 2015/2016. 12th ed. Berlin: Robert Koch Institute; 2020.

4. Kyle RA, Gertz MA, Witzig TE, et al. Review of 1027 patients with newly diagnosed multiple myeloma. Mayo Clin Proc. 2003;78(1):21-33. https://doi.org/ 10.4065/78.1.21.

5. Ninkovic S, Quach H. Shaping the treatment paradigm based on the current understanding of the pathobiology of multiple myeloma: an overview. Cancers (Basel). 2020;12(11):3488. https://doi.org/ 10.3390/cancers12113488.

6. Greipp PR, San Miguel J, Durie BG, et al. International staging system for multiple myeloma. J Clin Oncol. 2005;23(15):3412-20. https://doi.org/10. 1200/jco.2005.04.242.

7. Palumbo A, Avet-Loiseau H, Oliva S, et al. Revised international staging system for multiple myeloma: a report from International Myeloma Working Group. J Clin Oncol. 2015;33(26):2863-9. https:// doi.org/10.1200/jco.2015.61.2267.

8. Sonneveld P, Avet-Loiseau H, Lonial S, et al. Treatment of multiple myeloma with high-risk cytogenetics: a consensus of the International Myeloma Working Group. Blood. 2016;127(24):2955-62. https://doi.org/10.1182/blood-2016-01-631200.

9. Moreau P, San Miguel J, Sonneveld P, et al. Multiple myeloma: ESMO Clinical Practice Guidelines for diagnosis, treatment and follow-up. Ann Oncol. 2017;28:iv52-61. https://doi.org/10.1093/annonc/ mdx096.

10. Dimopoulos MA, Moreau P, Terpos E, et al. Multiple myeloma: EHA-ESMO Clinical Practice Guidelines for diagnosis, treatment and follow-up. Hemasphere. 2021;5(2): e528. https://doi.org/10.1097/ HS9.0000000000000528.

11. European Medicines Agency 2021. https://www. ema.europa.eu/en. Accessed 28 Oct 2021.

12. Steinmetz HT, Singh M, Lebioda A, et al. Patient characteristics and outcomes of relapsed/refractory multiple myeloma in patients treated with proteasome inhibitors in Germany. Oncol Res Treat. 2020;43(9):449-59. 000509018. 
13. Jagannath S, Abonour R, Durie BGM, et al. Heterogeneity of second-line treatment for patients with multiple myeloma in the Connect MM registry (2010-2016). Clin Lymphoma Myeloma Leuk. 2018;18(7):480-5. https://doi.org/10.1016/j.clml. 2018.04.007.

14. National Cancer Institute; 2020. The Surveillance, Epidemiology, and End Results (SEER) Program. Cancer Stat Facts: Myeloma. https://seer.cancer. gov/statfacts/html/mulmy.html. Accessed 28 Oct 2021.

15. Engelhardt M, Ihorst G, Singh $M$, et al. Real-world evaluation of health-related quality of life in patients with multiple myeloma from Germany. Clin Lymphoma Myeloma Leuk. 2021;21(2): e160-75. https://doi.org/10.1016/j.clml.2020.10. 002.

16. Yong K, Delforge M, Driessen C, et al. Multiple myeloma: patient outcomes in real-world practice. Br J Haematol. 2016;175(2):252-64. https://doi.org/ 10.1111/bjh.14213.

17. Durie BG, Harousseau JL, Miguel JS, et al. International uniform response criteria for multiple myeloma. Leukemia. 2006;20(9):1467-73. https://doi. org/10.1038/sj.leu.2404284.

18. Kyle RA, Rajkumar SV. Criteria for diagnosis, staging, risk stratification and response assessment of multiple myeloma. Leukemia. 2009;23(1):3-9. https://doi.org/10.1038/leu.2008.291.

19. Steinmetz TH, Singh M, Lebioda A, et al. Healthcare resource utilization and costs among patients with relapsed and/or refractory multiple myeloma treated with proteasome inhibitors in real-world clinical practice in Germany. J Med Econ. 2021;24(1): 114-22. https://doi.org/10.1080/13696998.2020. 1867469 .

20. Mohty M, Terpos E, Mateos MV, et al. Multiple myeloma treatment in real-world clinical practice: results of a prospective, multinational, noninterventional study. Clin Lymphoma Myeloma Leuk. 2018;18(10):e401-19. https://doi.org/10.1016/j. clml.2018.06.018.

21. Raab MS, Cavo M, Delforge M, et al. Multiple myeloma: practice patterns across Europe. $\mathrm{Br} \mathrm{J}$ Haematol. 2016;175(1):66-76. https://doi.org/10. 1111/bjh.14193.

22. Merz M, Kellermann L, Poenisch W, et al. Diagnosis and treatment of multiple myeloma in Germany: analysis of a nationwide multi-institutional survey. Ann Hematol. 2017;96(6):987-93. https://doi.org/ 10.1007/s00277-017-2991-0.
23. Verelst SGR, Blommestein HM, De Groot S, et al. Long-term outcomes in patients with multiple myeloma: a retrospective analysis of the Dutch Population-based HAematological Registry for Observational Studies (PHAROS). Hemasphere. 2018;2(4): e45. https://doi.org/10.1097/hs9. 0000000000000045 .

24. Despiégel N, Touboul C, Flinois A, et al. Healthrelated quality of life of patients with multiple myeloma treated in routine clinical practice in France. Clin Lymphoma Myeloma Leuk. 2019;19(1):e13-28. https://doi.org/10.1016/j.clml. 2018.08.019.

25. Merz M, Patel V, Kutikova L, et al. Treatment patterns in patients (PTS) with refractory/relapsed multiple myeloma (RRMM) in Germany between 2016 and 2018. Blood. 2019;134(suppl 1):4734. https://doi.org/10.1182/blood-2019-128030.

26. Chen J, Zhou L, Liu X, et al. Meta-analysis of clinical trials to assess denosumab over zoledronic acid in bone metastasis. Int J Clin Pharm. 2021;43(1): 2-10. https://doi.org/10.1007/s11096-020-01105-1.

27. Rasch S, Lund T, Asmussen JT, et al. Multiple myeloma associated bone disease. Cancers (Basel). 2020;12(8):2113. https://doi.org/10.3390/ cancers 12082113.

28. Kim C, Bhatta S, Cyprien L, Fonseca R, Hernandez $\mathrm{RK}$. Incidence of skeletal-related events among multiple myeloma patients in the United States at oncology clinics: observations from real-world data. J Bone Oncol. 2019;14: 100215. https://doi.org/10. 1016/j.jbo.2018.100215.

29. Rifkin RM, Abonour R, Terebelo H, et al. Connect MM Registry: the importance of establishing baseline disease characteristics. Clin Lymphoma Myeloma Leuk. 2015;15(6):368-76. https://doi.org/10. 1016/j.clml.2014.12.002.

30. Gonzalez-McQuire S, Dimopoulos MA, Weisel K, et al. Development of an initial conceptual model of multiple myeloma to support clinical and health economics decision making. MDM Policy Pract. 2019;4(1):2381468318814253. https://doi.org/10. $1177 / 2381468318814253$.

31. Saxe D, Seo EJ, Bergeron MB, Han JY. Recent advances in cytogenetic characterization of multiple myeloma. Int J Lab Hematol. 2019;41(1):5-14. https://doi.org/10.1111/ijlh.12882.

32. Knauf W, Kellermann L, Poenisch W, et al. How to treat multiple myeloma-a representative multicentre treatment survey. Onkologie. 2010;33(11): 604-10. https://doi.org/10.1159/000321124. 
33. Moehler TM, Merz M, Kellermann L, Goldschmidt H, Knauf W. Diagnostic and therapeutic approaches to multiple myeloma patients: "real-world" data from representative multicentre treatment surveys in Germany between 2008 and 2011. Oncol Lett. 2016;12(6):5043-51. https://doi.org/10.3892/ol. 2016.5375.

34. Oriol A, Steinmetz T, Patel V, et al. Clinical and treatment characteristics of patients with relapsed/ refractory multiple myeloma (RRMM) in Europe. Value Health. 2019;22:S497-8. https://doi.org/10. 1016/j.jval.2019.09.513.

35. Patel V, Kap EJ, Kutikova L. Treatment patterns in relapsed/refractory multiple myeloma patients in Germany. Value Health. 2019;22:S923. https://doi. org/10.1016/j.jval.2019.09.2740.

36. Raab MS, Fink L, Schoen P, et al. Evolution of multiple myeloma treatment practices in Europe from 2014 to 2016. Br J Haematol. 2019;18(5): 981-4. https://doi.org/10.1111/bjh.15680.

37. Bruno AS, Willson JL, Opalinska JM, et al. Recent real-world treatment patterns and outcomes in US patients with relapsed/refractory multiple myeloma. Expert Rev Hematol. 2020;13(9):1017-25. https://doi.org/10.1080/17474086.2020.1800451.

38. Chari A, Weisel KC, Usmani SZ, et al. Evolving treatment patterns in multiple myeloma (MM) differ by age and region: analysis from INSIGHT MM, a global, prospective, observational study. European Haematology Association 2019 congress abstract PF601. https://library.ehaweb.org/eha/2019/24th/ 266400/ajai.chari.evolving.treatment.patterns.in. multiple.myeloma.\%28mm\%29.differ.by.age.html. Accessed 24 Nov 2021.

39. Merz M, Vande Broek I, Pérez M, et al. Evolving treatment trends in relapsed/refractory multiple myeloma (RRMM) in Europe from 2016 to 2018: analysis of a multi-national survey. Blood. 2019;134(Suppl 1):3115. https://doi.org/10.1182/ blood-2019-122706.

40. de Azambuja E, Ameye L, Paesmans M, et al. The landscape of medical oncology in Europe by 2020. Ann Oncol. 2014;25(2):525-8. https://doi.org/10. 1093/annonc/mdt559. 